\title{
An eigenfunction expansion-variational method for the anti-plane electroelastic behavior of three-phase fiber composites
}

\author{
P. Yan ${ }^{a}$, C.P. Jiang ${ }^{a, b, *}$, F. Song ${ }^{b}$ \\ ${ }^{a}$ School of Aeronautic Science and Engineering, Beijing University of Aeronautics and Astronautics, 100191 Beijing, China \\ b State Key Laboratory of Nonlinear Mechanics (LNM), Institute of Mechanics, Chinese Academy of Sciences, 100190 Beijing, China
}

\section{A R T I C L E I N F O}

\section{Article history:}

Received 16 October 2010

Received in revised form 30 May 2011

Available online 28 June 2011

\section{Keywords:}

Effective electroelastic moduli

Interphase

Double period

Unit cell

Fiber-reinforced composites

\begin{abstract}
A B S T R A C T
The anti-plane electroelastic behavior of three-phase piezoelectric composites (fiber/interphase/matrix) with doubly periodic microstructures is dealt with. A new variational functional for a unit cell is constructed by incorporating the periodic boundary conditions into the energy functional. Then, by combining with the eigenfunction expansions of the complex potentials satisfying the fiber-interphase-matrix interfacial conditions, an eigenfunction expansion-variational method based on a unit cell is developed. The numerical results of the effective electroelastic moduli show a rapid convergence of the present method. A unified first-order approximation formula is also provided, where an equivalent parameter matrix reflecting the overall influence of the electroelastic properties of the fiber and interphase on the effective properties, is found. The equivalent parameter matrix can greatly simplify the complicated relation of the effective electroelastic properties to the internal structure of a three-phase fiber composite. Though the equivalent parameter matrix is extracted in the first-order approximation formula, its validity is also verified in the high-order numerical results.
\end{abstract}

(c) 2011 Elsevier Ltd. All rights reserved.

\section{Introduction}

Piezoelectric composites have been widely used in smart materials and structures, such as sensors and actuators, due to the outstanding performance compared with the monolithic piezoelectric materials.

In improving the toughness, flexibility and/or electroelastic performance, the two-phase composites have achieved great success. Many researches focused on this topic. For instance, Smith et al. (1985) found that the piezoelectric composites can provide a higher piezoelectric modulus than the constituents. The experiment and theoretical prediction by Chan and Unsworth (1989) showed that the PZT/polymer 1-3 composite has a comparable piezoelectric modulus with the monolithic PZT, while has a

\footnotetext{
* Corresponding author at: School of Aeronautic Science and Engineering, Beijing University of Aeronautics and Astronautics, 100191 Beijing, China. Tel.: +86 01082317508

E-mail address: jiangchiping@buaa.edu.cn (C.P. Jiang).
}

well-improved flexibility, if only the PZT volume fraction is up to 0.4 .

Three-phase piezoelectric composites may possess better designability compared with two-phase composites, and can serve as multifunctional materials. Lin and Sodano (2008) introduced a novel active piezoelectric structural fiber (the piezoceramics are coated onto a conductive structural fiber) that can be laid up in a composite to perform sensing and actuation, in addition to providing load bearing functionality. Beckert et al. (2001) reported a composite reinforced with hybrid fiber with an inactive core and a high performance piezoelectric coating, where the inactive core provides the mechanical support. Marcheselli and Venkatesh (2008) reported piezoelectric composites with hollow fibers, where the piezoelectric coupling constant may be enhanced significantly by the introduction of porosity. On the other hand, three-phase piezoelectric composites may be generated from two-phase piezoelectric composites by introducing an interphase between the inclusion phase and the matrix. Such a kind of interphase 
may be formed due to chemical reaction between the constituents, or be introduced to reduce high stress concentration at the interface arising from the mismatch between the material properties of constituents. And the stress concentrations may lead to failure of the piezoelectric composites.

Among researches about the topic of multi-phase piezoelectric material systems, some researches (Jiang and Cheung, 2001; Shen et al., 2009, 2005; Sudak, 2003; Yang and Gao, 2009) investigated electroelastic fields in the material systems of coated or multicoated inclusions in an infinite matrix, and some researches focused on the topic of effective properties of three-phase or multiphase piezoelectric composites. There are generally two kinds of models for solving the effective properties. One is the model of a multi-coated inhomogeneity embedded in an infinite matrix. Dinzart and Sabar (2009) solved the electroelastic coated inclusion problem based on the Green's functions technique and on the interfacial operators, and obtained the effective properties of composites through the Mori-Tanaka's model. Koutsawa et al. (2010) presented a micromechanics-based model to predict the effective thermo-electro-elastic properties of piezoelectric composite materials containing ellipsoidal multi-coated inhomogeneities. Lin and Sodano (2010) extended the double inclusion model to multiphase composites with piezoelectric constituents for studying a novel active structural fiber composite introduced by them. These three works presented general analytical solutions for effective piezoelectric properties by treating the inclusion interactions either approximately or in a statistical sense. The other is periodic microstructure model. Beckert et al. (2001), Marcheselli and Venkatesh (2008), and Lin and Sodano (2008) gave finite element solutions of effective properties for the three-phase composites with periodic microstructure. Guinovart-Díaz et al. (2008) determined effective moduli for three-phase piezoelectric unidirectional composites by using asymptotic homogenization scheme for periodic media. More recently, Hashemi et al. (2010) developed a robust homogenization scheme for determination of the effective properties of a periodic piezoelectric composite with general multi-coated inhomogeneities, by combining the Fourier series expansion approach with the eigen-field concept.

Though some very general schemes (such as Koutsawa et al., 2010; Hashemi et al., 2010) for determination of the effective properties of the multi-phase piezoelectric composites have been presented, unified and convenient engineering closed-form formulae of effective properties are still desirable for the design of piezoelectric composites. Moreover, the existing researches show that the effective properties are complicatedly related to a large number of microstructural parameters, such as the properties and arrangements of constituents and the thickness of interphase. Therefore, it is still necessary to simplify such complicated relations by extracting equivalent parameters. Additionally, some piezoelectric composites possess periodic microstructures, and the periodic composite models (Berger et al., 2006; Bravo-Castillero et al., 2001; Rodríguez-Ramos et al., 2010; Nemat-Nasser and Hori, 1999; Pettermann and Suresh, 2000; Xia et al., 2003; Xu et al., 2007; Yu and Somphone, 2009) provide useful limiting values of interacting inclusions from entirely disorder (random) to order. Especially, the design of an advanced composite is generally the one for a unit cell (Sun et al., 2001). Thus, it is valuable to develop a new analytical method for analysis of the composites with periodic microstructures.

The present work is devoted to develop an eigenfunction expansion-variational method for analysis of the anti-plane electroelastic behavior of three-phase piezoelectric composites (fiber/interphase/matrix) with a doubly periodic microstructure. High-order numerical solution and a convenient engineering closed-form formula for the effective electroelastic moduli are presented. In addition, an equivalent parameter matrix reflecting the overall influence of the electroelastic properties of the fiber and interphase on the effective moduli, and a parameter reflecting the fiber distribution are extracted in the closed-form formula. The validity and accuracy of these parameters are verified.

\section{Statement and formulation of the problem}

As shown in Fig. 1(a), a piezoelectric material is subjected to combined antiplane shear and inplane $\left(O x_{1} x_{2}-\right.$ plane) electrical loads, with a poling direction being along the $x_{3}$-axis. Then only the antiplane displacement $w$ and inplane electrical potential $\varphi$ need to be considered, and they are the functions of $x_{1}$ and $x_{2}$ only,

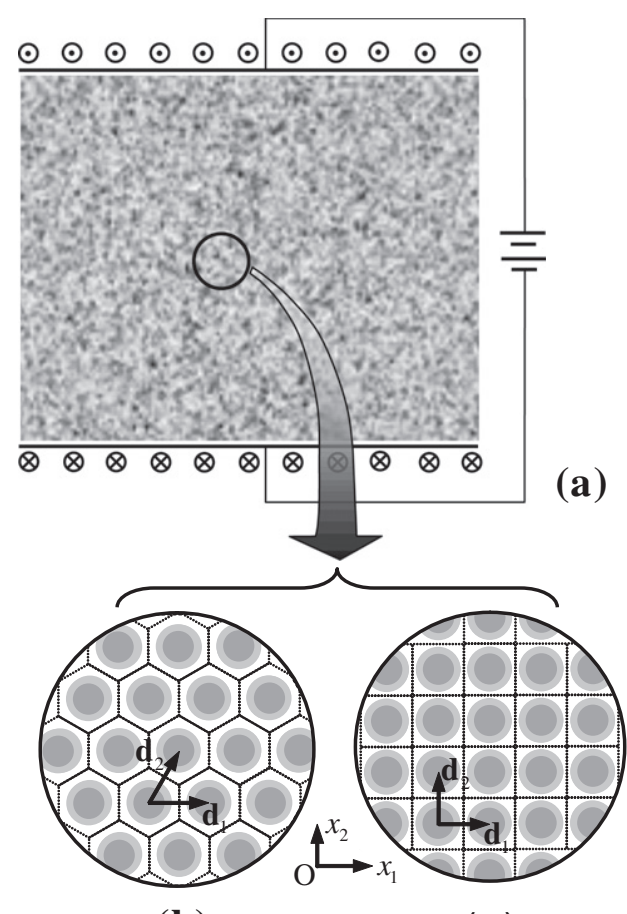

(b)

(c)

Fig. 1. Cross section of a three-phase piezoelectric fiber composite and its two kinds of doubly periodic microstructures. (a) The composite is subjected to combined antiplane shear and inplane ( $O x_{1} x_{2}$-plane) electrical loads; (b) hexagonal fiber array; (c) square fiber array. 
$\left[\begin{array}{l}w \\ \varphi\end{array}\right]=\left[\begin{array}{l}w\left(x_{1}, x_{2}\right) \\ \varphi\left(x_{1}, x_{2}\right)\end{array}\right]$

The strain and electrical field components $\varepsilon_{i 3}$ and $E_{i}$ $(i=1,2)$ satisfy the gradient equations:

$\left[\begin{array}{c}2 \varepsilon_{13} \\ -E_{1}\end{array}\right]=\left[\begin{array}{l}\frac{\partial w}{\partial x_{1}} \\ \frac{\partial \varphi}{\partial x_{1}}\end{array}\right], \quad\left[\begin{array}{c}2 \varepsilon_{23} \\ -E_{2}\end{array}\right]=\left[\begin{array}{l}\frac{\partial w}{\partial x_{2}} \\ \frac{\partial \varphi}{\partial x_{2}}\end{array}\right]$

For a transversely isotropic piezoelectric material, say, the fiber, interphase and matrix, the antiplane constitutive equations (Tiersten, 1969; Tong et al., 2006) can be written as:

$\left[\begin{array}{c}\tau_{i 3} \\ D_{i}\end{array}\right]=\left[\begin{array}{cc}C_{44} & e_{15} \\ e_{15} & -\kappa_{11}\end{array}\right]\left[\begin{array}{c}2 \varepsilon_{i 3} \\ -E_{i}\end{array}\right]$

where $\tau_{i 3}, D_{i}(i=1,2)$ are the antiplane shear stress and inplane electrical displacement components, respectively; $C_{44}, e_{15}$ and $\kappa_{11}$ are the shear, piezoelectric and dielectric moduli of the material, respectively.

The equilibrium equations and charge equations are

$\sum_{i=1}^{2} \frac{\partial \tau_{i 3}}{x_{i}}=0, \quad \sum_{i=1}^{2} \frac{\partial D_{i}}{x_{i}}=0$

For brevity and convenience, now introduce matrix notations for the variables as follows:

$\mathbf{w}=\left[\begin{array}{l}w \\ \varphi\end{array}\right], \quad \gamma_{1}=\left[\begin{array}{c}2 \varepsilon_{13} \\ -E_{1}\end{array}\right], \quad \gamma_{2}=\left[\begin{array}{c}2 \varepsilon_{23} \\ -E_{2}\end{array}\right]$,

$\boldsymbol{\gamma}=\left[\begin{array}{cc}2 \varepsilon_{13} & 2 \varepsilon_{23} \\ -E_{1} & -E_{2}\end{array}\right], \quad \tau_{1}=\left[\begin{array}{c}\tau_{13} \\ D_{1}\end{array}\right], \quad \tau_{2}=\left[\begin{array}{c}\tau_{23} \\ D_{2}\end{array}\right]$,

$\tau=\left[\begin{array}{ll}\tau_{13} & \tau_{23} \\ D_{1} & D_{2}\end{array}\right], \nabla=\left[\frac{\partial}{\partial x_{1}}, \frac{\partial}{\partial x_{2}}\right]$,

$\mathbf{x}=\left[\begin{array}{l}x_{1} \\ x_{2}\end{array}\right], \quad \mathbf{L}=\left[\begin{array}{cc}C_{44} & e_{15} \\ e_{15} & -\kappa_{11}\end{array}\right]$

where $\mathbf{w}, \gamma$ and $\tau$ are called generalized displacement, strain and stress, respectively. Then the basic equations are put in matrix form:

gradient equation : $\gamma=\mathbf{w} \otimes \nabla$

constitutive equation : $\tau=\mathbf{L} \boldsymbol{\gamma}$

equilibrium equation : $\nabla \tau^{\mathrm{T}}=\mathbf{0}$

where superscript " $T$ " denotes transpose.

From Eqs. (6a)-(6c), the displacement and electric potential fields satisfy the following Laplace's equation:

$\nabla^{2} \mathbf{w}=\mathbf{0}$

and $\nabla^{2}\left(=\frac{\partial^{2}}{x_{1}^{2}}+\frac{\partial^{2}}{x_{2}^{2}}\right)$ is the Laplacian operator.

\section{Periodicity conditions and variational functional for a unit cell}

Consider a piezoelectric composite with a doubly periodic microstructure as shown in Fig. 1. Two fiber distributions of practical importance, i.e., the hexagonal and square arrays, are shown in Fig. 1(b) and (c), respectively, where $\mathbf{d}_{1}$ and $\mathbf{d}_{2}$ denote two fundamental periods. A general period $\mathbf{p}$ can be defined as: $\mathbf{p}=m_{1} \mathbf{d}_{1}+m_{2} \mathbf{d}_{2}$

where $m_{1}$ and $m_{2}$ are arbitrary integers. For the piezoelectric behavior considered here, the displacement field $w(\mathbf{x})$ and electrical potential field $\varphi(\mathbf{x})$ are quasi-periodic, the stress field $\left\{\tau_{13}(\mathbf{x}), \tau_{23}(\mathbf{x})\right\}$ and electrical displacement field $\left\{D_{1}(\mathbf{x}), D_{2}(\mathbf{x})\right\}$ are periodic, and they can be expressed as:

$$
\left\{\begin{array}{l}
\mathbf{w}(\mathbf{x}+\mathbf{p})-\mathbf{w}(\mathbf{x})=\langle\gamma\rangle \mathbf{p} \\
\tau(\mathbf{x}+\mathbf{p})-\tau(\mathbf{x})=\mathbf{0}
\end{array}\right.
$$

where $\langle\boldsymbol{\gamma}\rangle$ denotes the average of the strain and electrical field matrix $\gamma$ over a unit cell.

Due to the periodicity, unit cells are picked out for analysis, and the hexagonal and square unit cells are shown in Fig. 2(a) and (b), respectively. The unit cell boundaries can be divided into $\partial V^{+}=\sum_{s} \partial V_{s}^{+}$and $\partial V^{-}=\sum_{s} \partial V_{s}^{-}$, where $s=1,2,3$ in Fig. 2(a) and $s=1,2$ in Fig. 2(b). By a proper translation $\mathbf{p}^{s}$, the boundary $\partial V_{s}^{-}$will coincide with the boundary $\partial V_{s}^{+}$, where $\mathbf{p}^{1}=\mathbf{d}_{1}, \mathbf{p}^{2}=\mathbf{d}_{2}$ and $\mathbf{p}^{3}=\mathbf{d}_{2}-\mathbf{d}_{1}$ in Fig. 2(a) and $\mathbf{p}^{1}=\mathbf{d}_{1}$ and $\mathbf{p}^{2}=\mathbf{d}_{2}$ in Fig. 2(b). Then the periodic boundary conditions of a unit cell corresponding to Eq. (9) can be written as:

$$
\left\{\begin{array}{l}
\mathbf{w}^{s+}-\mathbf{w}^{s-}=\langle\gamma\rangle \mathbf{p}^{s} \\
\mathbf{t}^{s+}+\mathbf{t}^{s-}=\mathbf{0}
\end{array}\right.
$$

where $\mathbf{t}(=\tau \mathbf{n})$ denotes the generalized boundary stress consisting of the boundary stress and boundary electrical displacement. $\mathbf{n}$ denotes the unit normal vector on the boundary; the quantities with superscripts " $s-$ " and " $s+$ " are corresponding to taking values from $\partial V_{s}^{-}$and $\partial V_{s}^{+}$, respectively.

By using the Lagrangian multiplier method, the periodic boundary conditions of a unit cell can be incorporated into the functional for the piezoelectric issue under consideration:

$\Pi=\int_{V} \frac{1}{2} \tau: \gamma d V+\int_{\partial V^{+}} \lambda^{*} \cdot\left(\mathbf{w}^{+}-\mathbf{w}^{-}-\langle\boldsymbol{\gamma}\rangle \mathbf{p}\right) d S$

where $\lambda^{*}$ is a Lagrangian multiplier to be determined. The variation of the generalized functional (11) can be written as:

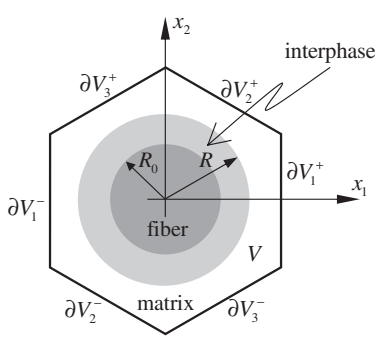

(a)

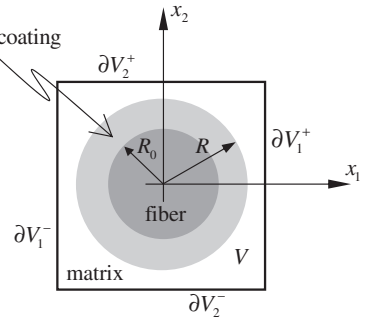

(b)
Fig. 2. Two kinds of unit cells taken from three-phase fiber composites (fiber/interphase/matrix). (a) Hexagonal unit cell and (b) square unit cell. 


$$
\begin{aligned}
\delta \Pi= & \int_{V} \boldsymbol{\tau}: \delta \gamma d V+\int_{\partial V^{+}} \delta \lambda^{*} \cdot\left(\mathbf{w}^{+}-\mathbf{w}^{-}-\langle\gamma\rangle \mathbf{p}\right) d S \\
& +\int_{\partial V^{+}} \lambda^{*} \cdot\left(\delta \mathbf{w}^{+}-\delta \mathbf{w}^{-}\right) d S=-\int_{V}\left(\nabla \tau^{\mathrm{T}}\right) \cdot \delta \mathbf{w} d V \\
& +\int_{\partial V} \mathbf{t} \cdot \delta \mathbf{w} d S+\int_{\partial V^{+}} \delta \lambda^{*} \cdot\left(\mathbf{w}^{+}-\mathbf{w}^{-}-\langle\gamma\rangle \mathbf{p}\right) d S \\
& +\int_{\partial V^{+}} \lambda^{*} \cdot\left(\delta \mathbf{w}^{+}-\delta \mathbf{w}^{-}\right) d S
\end{aligned}
$$

where $\delta(\cdot)$ denotes the variation, and the second term can be written as:

$\int_{\partial V} \mathbf{t} \cdot \delta \mathbf{w} d S=\int_{\partial V^{+}}\left(\mathbf{t}^{+} \cdot \delta \mathbf{w}^{+}+\mathbf{t}^{-} \cdot \delta \mathbf{w}^{-}\right) d S$

If the equilibrium equation (Eq. (6a)) is satisfied in advance, the variation (12) can be written as:

$$
\begin{aligned}
\delta \Pi= & \int_{\partial V^{+}} \delta \lambda^{*} \cdot\left(\mathbf{w}^{+}-\mathbf{w}^{-}-\langle\gamma\rangle \mathbf{p}\right) d S+\int_{\partial V^{+}}\left(\mathbf{t}^{-}-\lambda^{*}\right) \cdot \delta \mathbf{w}^{-} d S \\
& +\int_{\partial V^{+}}\left(\mathbf{t}^{+}+\lambda^{*}\right) \cdot \delta \mathbf{w}^{+} d S
\end{aligned}
$$

It can be seen form Eq. (14) that the stationary condition requires:

$$
\mathbf{w}^{+}-\mathbf{w}^{-}-\langle\gamma\rangle \mathbf{p}=\mathbf{0}, \quad \mathbf{t}^{+}+\mathbf{t}^{-}=\mathbf{0}, \quad \lambda^{*}=-\mathbf{t}^{+}
$$

Thus, the Lagrangian multiplier $\lambda^{*}$ is determined as $-\mathbf{t}^{+}$, and the variation of the generalized functional (14) can be rewritten as:

$$
\begin{aligned}
\delta \Pi= & -\int_{\partial V^{+}} \delta \mathbf{t}^{+} \cdot\left(\mathbf{w}^{+}-\mathbf{w}^{-}-\langle\gamma\rangle \mathbf{p}\right) d S \\
& +\int_{\partial V^{+}}\left(\mathbf{t}^{+}+\mathbf{t}^{-}\right) \cdot \delta \mathbf{w}^{-} d S
\end{aligned}
$$

The stationary condition $\delta \Pi=0$ leads to

$$
\begin{aligned}
& \int_{\partial V^{+}} \delta \mathbf{t}^{+} \cdot\left(\mathbf{w}^{+}-\mathbf{w}^{-}\right) d S-\int_{\partial V^{+}}\left(\mathbf{t}^{+}+\mathbf{t}^{-}\right) \cdot \delta \mathbf{w}^{-} d S \\
& \quad=\int_{\partial V^{+}} \delta \mathbf{t}^{+} \cdot\langle\boldsymbol{\gamma}\rangle \mathbf{p} d S
\end{aligned}
$$

or

$$
\begin{aligned}
& \sum_{S} \int_{\partial V_{s}^{+}} \delta \mathbf{t}^{S+} \cdot\left(\mathbf{w}^{S+}-\mathbf{w}^{S-}\right) d S-\sum_{S} \int_{\partial V_{s}^{+}}\left(\mathbf{t}^{S+}+\mathbf{t}^{S-}\right) \cdot \delta \mathbf{w}^{S-} d S \\
& =\sum_{S} \int_{\partial V_{s}^{+}} \delta \mathbf{t}^{S+} \cdot\langle\gamma\rangle \mathbf{p}^{S} d S
\end{aligned}
$$

in another form. This stationary condition (18) will be used to develop an eigenfunction expansion-variational method based on a unit cell.

\section{Eigenfunction expansions of complex potentials}

\subsection{Complex potential}

Because the generalized displacement satisfies the Laplace's equation (7), and from Eqs. (6a)-(6c), the generalized displacement $\mathbf{w}$, generalized stress $\tau$ and generalized resultant force $\mathbf{T}$ can be formulated by two potentials $\left\{f_{1}(z), f_{2}(z)\right\}$ with a vector form $\mathbf{f}(z)$ :

$$
\begin{aligned}
& \mathbf{w}=\frac{1}{2}[\mathbf{f}(z)+\overline{\mathbf{f}(z)}] \\
& \tau_{1}-\mathrm{i} \tau_{2}=\mathbf{L f}^{\prime}(z) \\
& \mathbf{T}=\int_{\mathrm{A}}^{\mathrm{B}} \tau \mathbf{n} d S=\frac{1}{2 \mathrm{i}} \mathbf{L}[\mathbf{f}(z)-\overline{\mathbf{f}(z)}]_{\mathrm{A}}^{\mathrm{B}}
\end{aligned}
$$

where, $z=x_{1}+i x_{2}$ is a complex variable, the over bar denotes the complex conjugate, the prime denotes the derivative with respect to $z,[\cdot]_{A}^{B}$ denotes the difference of the values of the bracketed function from point $A$ to point B.

\subsection{Eigenfunction expansions in a unit cell}

A typical unit cell of a three-phase fiber composite with a doubly periodic microstructure is divided into three regions occupied, respectively, by a fiber, a coating/interphase and a surrounding matrix as shown in Fig. 2. $R_{0}$ and $R$ are the internal and external radii of the coating/ interphase, respectively. Subscripts/superscripts "f", "c" and " $\mathrm{m}$ " refer to the fiber, coating/interphase and matrix, respectively.

The complex potential $\mathbf{f}_{\mathrm{f}}(z)$ in the fiber region can be expanded into a Taylor series, $\mathbf{f}_{\mathrm{c}}(z)$ in the coating/interphase region and $\mathbf{f}_{\mathrm{m}}(z)$ in the matrix region can be expanded into Laurent series,

$$
\begin{aligned}
& \mathbf{f}_{\mathrm{f}}(z)=\sum_{n=1}^{\infty} \mathbf{C}_{1 n} z^{2 n-1} \\
& \mathbf{f}_{\mathrm{c}}(z)=\sum_{n=1}^{\infty} \mathbf{C}_{2 n} z^{-(2 n-1)}+\sum_{n=1}^{\infty} \mathbf{C}_{3 n} z^{2 n-1} \\
& \mathbf{f}_{\mathrm{m}}(z)=\sum_{n=1}^{\infty} \mathbf{C}_{4 n} z^{-(2 n-1)}+\sum_{n=1}^{\infty} \mathbf{C}_{5 n} z^{2 n-1}
\end{aligned}
$$

where $\mathbf{C}_{1 n}, \mathbf{C}_{2 n}, \mathbf{C}_{3 n}, \mathbf{C}_{4 n}$ and $\mathbf{C}_{5 n}$ are complex coefficient vectors. Due to the centrosymmetry of the unit cell, only odd terms in Eqs. (20a)-(20c) remain.

Now examine the relations between the five sets of unknown coefficients.

The continuity conditions of the generalized resultant force $\mathbf{T}$ and the generalized displacement $\mathbf{w}$ across the fiber-coating and coating-matrix interfaces can be written as

$\mathbf{T}_{\mathrm{f}}=\mathbf{T}_{\mathrm{c}}, \quad \mathbf{w}_{\mathrm{f}}=\mathbf{w}_{\mathrm{c}} \quad$ at $\quad|z|=R_{0}$

$\mathbf{T}_{\mathrm{c}}=\mathbf{T}_{\mathrm{m}}, \quad \mathbf{w}_{\mathrm{c}}=\mathbf{w}_{\mathrm{m}} \quad$ at $\quad|z|=R$

The interfacial conditions (21) can provide four sets of equations with respect to five sets of unknown complex coefficients $\mathbf{C}_{1 n}, \mathbf{C}_{2 n}, \mathbf{C}_{3 n}, \mathbf{C}_{4 n}$ and $\mathbf{C}_{5 n}$. Only one set of independent unknown complex coefficients (choose $\mathbf{C}_{5 n}$ ) remains. It will be seen that the estimation of the effective electroelastic moduli requires only $\mathbf{f}_{\mathrm{m}}(z)$. Substituting Eqs. (20a)-(20c) into Eqs. (19a) and (19c), and then into Eqs. (21a) and (21b) as detailed in Appendix A, one obtains the relation between $\mathbf{C}_{4 n}$ and $\mathbf{C}_{5 n}$,

$\mathbf{C}_{4 n}=\boldsymbol{\eta}_{n} R^{4 n-2} \overline{\mathbf{C}}_{5 n}$ 
where

$\boldsymbol{\eta}_{n}=\left[\mathbf{I}+\left(\mathbf{I}+\mathbf{L}_{\mathrm{c}}^{-1} \mathbf{L}_{\mathrm{m}}\right)^{-1} \boldsymbol{\eta}_{\mathrm{fc}}\left(\mathbf{L}_{\mathrm{c}}^{-1} \mathbf{L}_{\mathrm{m}}-\mathbf{I}\right)\left(R_{0} / R\right)^{4 n-2}\right]^{-1}$

$\cdot\left[\left(\mathbf{I}+\mathbf{L}_{\mathrm{c}}^{-1} \mathbf{L}_{\mathrm{m}}\right)^{-1} \boldsymbol{\eta}_{\mathrm{fc}}\left(\mathbf{I}+\mathbf{L}_{\mathrm{c}}^{-1} \mathbf{L}_{\mathrm{m}}\right)\left(R_{0} / R\right)^{4 n-2}+\boldsymbol{\eta}_{\mathrm{cm}}\right]$

$\boldsymbol{\eta}_{\mathrm{fc}}=\left(\mathbf{L}_{\mathrm{f}}+\mathbf{L}_{\mathrm{c}}\right)^{-1}\left(\mathbf{L}_{\mathrm{c}}-\mathbf{L}_{\mathrm{f}}\right)$

$\boldsymbol{\eta}_{\mathrm{cm}}=\left(\mathbf{L}_{\mathrm{c}}^{-1} \mathbf{L}_{\mathrm{m}}+\mathbf{I}\right)^{-1}\left(\mathbf{L}_{\mathrm{c}}^{-1} \mathbf{L}_{\mathrm{m}}-\mathbf{I}\right)$

and $\mathbf{I}$ is an identity matrix. Then, the eigenfunction expansion of the complex potential $\mathbf{f}_{\mathrm{m}}(z)$ can be written as:

$\mathbf{f}_{\mathrm{m}}(z)=\sum_{n=1}^{\infty} \boldsymbol{\eta}_{n} R^{4 n-2} \overline{\mathbf{C}}_{5 n} z^{-(2 n-1)}+\mathbf{C}_{5 n} z^{2 n-1}$

Consider the square and hexagonal unit cells shown in Fig. 2. If the boundary conditions are also symmetric about $x_{2}$ axis, the expansion coefficients additionally satisfy following relations:

$\mathbf{C}_{1 n}=-\overline{\mathbf{C}}_{1 n}, \quad \mathbf{C}_{2 n}=-\overline{\mathbf{C}}_{2 n}, \quad \mathbf{C}_{3 n}=-\overline{\mathbf{C}}_{3 n}$,

$\mathbf{C}_{4 n}=-\overline{\mathbf{C}}_{4 n}, \quad \mathbf{C}_{5 n}=-\overline{\mathbf{C}}_{5 n}$

That is, the expansion coefficients are pure imaginary. The eigenfunction expansion of the complex potential $\mathbf{f}_{\mathrm{m}}(z)$ is further simplified as:

$\mathbf{f}_{\mathrm{m}}(z)=\sum_{n=1}^{\infty}\left(-\boldsymbol{\eta}_{n} R^{4 n-2} z^{-(2 n-1)}+\mathbf{I} z^{2 n-1}\right) \mathbf{C}_{5 n}$

The remaining work is to determine one set of unknown coefficient, $\mathbf{C}_{5 n}$, which can be completed by using the stationary condition (18).

\subsection{Determination of the unknown coefficients}

In this section, a detailed solving procedure of the unknown coefficients is given for the case of the shear stress field and electrical displacement field being symmetric about $x_{2}$ axis. Substituting Eq. (28) into Eqs. (19a)-(19c), and taking an appropriate truncation of each expansion, the generalized stresses, displacement, boundary stress and resultant force can be expressed as follows:

$\tau_{i}=\sum_{n=1}^{N} \tau_{i}^{(n)} \mathbf{X}_{n}, \quad \mathbf{w}=\sum_{n=1}^{N} \mathbf{w}^{(n)} \mathbf{X}_{n}$

$\mathbf{t}=\sum_{n=1}^{N} \mathbf{t}^{(n)} \mathbf{X}_{n}, \quad \mathbf{T}=\sum_{n=1}^{N} \mathbf{T}^{(n)} \mathbf{X}_{n}, \quad i=1,2$

where

$$
\left\{\begin{array}{l}
\mathbf{X}_{n}=\mathbf{i}_{5 n} \\
\tau_{1}^{(n)}=\frac{1}{2 \mathrm{i}} \mathbf{L}_{\mathrm{m}}\left[\mathbf{I}(2 n-1)\left(z^{2 n-2}-\bar{z}^{2 n-2}\right)\right. \\
\left.\quad+\boldsymbol{\eta}_{n} R^{4 n-2}(1-2 n)\left(\bar{z}^{-2 n}-z^{-2 n}\right)\right] \\
\tau_{2}^{(n)}=\frac{1}{2} \mathbf{L}_{\mathrm{m}}\left[\mathbf{I}(2 n-1)\left(z^{2 n-2}+\bar{z}^{2 n-2}\right)\right. \\
\left.\quad-\boldsymbol{\eta}_{n} R^{4 n-2}(1-2 n)\left(\bar{z}^{-2 n}+z^{-2 n}\right)\right] \\
\quad \mathbf{w}^{(n)}=\frac{1}{2 \mathrm{i}}\left[\mathbf{I}\left(z^{2 n-1}-\bar{z}^{2 n-1}\right)+\boldsymbol{\eta}_{n} R^{4 n-2}\left(\bar{z}^{1-2 n}-z^{1-2 n}\right)\right] \\
\mathbf{t}^{(n)}=\boldsymbol{\tau}^{(n)} \mathbf{n} \\
\mathbf{T}^{(n)}=-\frac{1}{2} \mathbf{L}_{\mathrm{m}}\left[\mathbf{I}\left(z^{2 n-1}+\bar{z}^{2 n-1}\right)-\boldsymbol{\eta}_{n} R^{4 n-2}\left(\bar{z}^{1-2 n}+z^{1-2 n}\right)\right]_{\mathrm{A}}^{\mathrm{B}}
\end{array}\right.
$$$$
1 \leqslant n \leqslant N
$$$$
1 \leqslant n \leqslant N
$$$$
1 \leqslant n \leqslant N
$$$$
1 \leqslant n \leqslant N
$$$$
1 \leqslant n \leqslant N
$$$$
1 \leqslant n \leqslant N
$$

The substitution of Eq. (29) into the stationary condition (18) yields the following linear algebraic equations:

$\sum_{m=1}^{N} \mathbf{A}_{n m} \mathbf{X}_{m}=\mathbf{B}_{n}, \quad n=1,2, \ldots, N$

where

$$
\begin{aligned}
\mathbf{A}_{n m}= & \sum_{s} \int_{\partial V_{s}^{+}}\left(\mathbf{t}_{(n)}^{s+}\right)^{\mathrm{T}}\left(\mathbf{w}_{(m)}^{s+}-\mathbf{w}_{(m)}^{s-}\right) d S \\
& -\sum_{s} \int_{\partial V_{s}^{+}}\left(\mathbf{w}_{(n)}^{s-}\right)^{\mathrm{T}}\left(\mathbf{t}_{(m)}^{s+}+\mathbf{t}_{(m)}^{s-}\right) d S \\
\mathbf{B}_{n}= & \sum_{S} \int_{\partial V_{s}^{+}}\left(\mathbf{t}_{(n)}^{s+}\right)^{\mathrm{T}}\left(\langle\boldsymbol{\gamma}\rangle \mathbf{p}^{s}\right) d S=\sum_{S}\left(\mathbf{T}_{(n)}^{s+}\right)^{\mathrm{T}}\left(\langle\boldsymbol{\gamma}\rangle \mathbf{p}^{S}\right)
\end{aligned}
$$

$\mathbf{t}_{(n)}^{s+}, \mathbf{w}_{(m)}^{s+}$ and $\mathbf{T}_{(n)}^{s+}$ denote taking the values of $\mathbf{t}^{(n)}, \mathbf{w}^{(m)}$ and $\mathbf{T}^{(n)}$ from $\partial V_{s}^{+}$, respectively; and the quantities with the superscript "s-" are corresponding to taking values from $\partial V_{s}^{-}$.

Once the unknown coefficients are determined by Eqs. (31a)-(31c), the generalized stress and displacement fields can be obtained by Eqs. (29) and (30).

\section{Effective electroelastic moduli}

The effective electroelastic moduli of a composite, $\mathbf{L}_{i j}^{\mathrm{e}}$, are determined with the aid of the average field theory (Nemat-Nasser and Hori, 1999):

$\left\langle\tau_{i}\right\rangle=\mathbf{L}_{i j}^{\mathrm{e}}\left\langle\gamma_{j}\right\rangle, \quad i, j=1,2$

where $\left\langle\tau_{i}\right\rangle$ is the average generalized stress within the unit cell, which can be calculated by the following formula:

$\left\langle\tau_{i}\right\rangle=\frac{1}{V} \int_{V} \tau_{i} d V=\frac{1}{V} \sum_{s} \mathbf{T}^{s+} p_{i}^{s}$

$\mathbf{T}^{s+}$ is the generalized resultant force on the boundary $\partial V_{s}^{+}$. It is worth noting that the periodic boundary conditions (10) are prescribed for the unit cell by setting the average generalized strain $\langle\gamma\rangle$, and then the average generalized stress $\langle\tau\rangle$ is solved for calculating the effective electroelastic moduli.

For a piezoelectric composite with a square or hexagonal array of fibers, according to the symmetry, the effective electroelastic moduli matrix satisfies: 
$\mathbf{L}_{11}^{\mathrm{e}}=\mathbf{L}_{22}^{\mathrm{e}}=\mathbf{L}_{\mathrm{e}}=\left[\begin{array}{cc}C_{44}^{\mathrm{e}} & e_{15}^{\mathrm{e}} \\ e_{15}^{\mathrm{e}} & -\kappa_{11}^{\mathrm{e}}\end{array}\right], \quad \mathbf{L}_{12}^{\mathrm{e}}=\mathbf{L}_{21}^{\mathrm{e}}=\mathbf{0}$

In order to determine the reduced effective electroelastic moduli matrix $\mathbf{L}_{\mathrm{e}}$, a boundary condition symmetric about $x_{2}$ axis are prescribed for the symmetric unit cell (as shown in Fig. $1(\mathrm{a})$ ) by setting $\left\langle\gamma_{1}\right\rangle=\mathbf{0}$ and $\left\langle\gamma_{2}\right\rangle \neq \mathbf{0}$. Then the average generalized stress can be calculated by:

$\left\langle\tau_{2}\right\rangle=\frac{1}{V} \sum_{n=1}^{N} \sum_{m=1}^{N}\left(\sum_{s} \mathbf{T}_{(n)}^{s+} p_{2}^{s}\right)\left(\mathbf{A}^{-1}\right)_{n m}\left(\sum_{s} \mathbf{T}_{(m)}^{s+} p_{2}^{s}\right)\left\langle\gamma_{2}\right\rangle$

From Eqs. (32) and (35), it is obtained that

$\mathbf{L}_{\mathrm{e}}=\mathbf{L}_{22}^{\mathrm{e}}=\frac{1}{V} \sum_{n=1}^{N} \sum_{m=1}^{N}\left(\sum_{s} \mathbf{T}_{(n)}^{s+} p_{2}^{s}\right)\left(\mathbf{A}^{-1}\right)_{n m}\left(\sum_{s} \mathbf{T}_{(m)}^{s+} p_{2}^{s}\right)$

When the term number $N$ of the eigenfunction expansion is large enough, high-order numerical results of the effective electroelastic moduli are obtained. When $N=1$, a first-order approximation formula is obtained. Interestingly, as detailed in Appendix B, for the square and hexagonal fiber arrays, the first-order approximation formula can be written as a unified expression:

$\mathbf{L}_{\mathrm{e}}=\mathbf{L}_{\mathrm{m}}(\pi \mathbf{I}-\alpha \lambda \boldsymbol{\eta})^{2}\left[\pi^{2} \mathbf{I}+\left(2 \pi^{2}-2 \alpha \pi\right) \lambda \boldsymbol{\eta}-\alpha^{2} \lambda^{2} \boldsymbol{\eta}^{2}\right]^{-1}$

where $\lambda$ is a total volume fraction of the fiber and coating/ interphase; and $\boldsymbol{\eta}$ is a composite parameter matrix synthetically characterizing the constituent properties and relative coating/interphase thickness,

$\boldsymbol{\eta}=\left[\mathbf{I}+\left(\mathbf{I}+\mathbf{L}_{\mathrm{c}}^{-1} \mathbf{L}_{\mathrm{m}}\right)^{-1} \boldsymbol{\eta}_{\mathrm{fc}}\left(\mathbf{L}_{\mathrm{c}}^{-1} \mathbf{L}_{\mathrm{m}}-\mathbf{I}\right)\left(R_{0} / R\right)^{2}\right]^{-1}$

$$
\cdot\left[\left(\mathbf{I}+\mathbf{L}_{\mathrm{c}}^{-1} \mathbf{L}_{\mathrm{m}}\right)^{-1} \boldsymbol{\eta}_{\mathrm{fc}}\left(\mathbf{I}+\mathbf{L}_{\mathrm{c}}^{-1} \mathbf{L}_{\mathrm{m}}\right)\left(R_{0} / R\right)^{2}+\boldsymbol{\eta}_{\mathrm{cm}}\right]
$$

$\boldsymbol{\eta}_{\mathrm{fc}}$ and $\boldsymbol{\eta}_{\mathrm{cm}}$ are given in Eqs. (24) and (25), and $\alpha$ is a parameter reflecting the distribution of fibers,

$\alpha= \begin{cases}2 & \text { for square array } \\ 3 \sqrt{3} / 2 & \text { for hexagonal array }\end{cases}$

It is interesting to note that when $\alpha=\pi$, the first-order approximation formula (37) can be reduced into the result of the generalized self-consistent method (Jiang and Cheung, 2001; Jiang et al., 2001):

$\mathbf{L}_{\mathrm{e}}=\mathbf{L}_{\mathrm{m}}(\mathbf{I}-\lambda \boldsymbol{\eta})(\mathbf{I}+\lambda \boldsymbol{\eta})^{-1}$

For a two-phase fiber reinforced piezoelectric composite, this formula coincides with the result in Jiang and Cheung (2001).

The numerical results in the next section will show that the first-order approximation formula can consider the effect of the fiber distribution to some extent, thus can be regarded as an improvement of the generalized selfconsistent formula (40).

From the first-order approximation formula (37) and the generalized self-consistent formula (40), an equivalent parameter matrix, $\boldsymbol{\eta}$, reflecting the overall influence of the electroelastic properties of the fiber and interphase on the effective moduli, is found. Such an equivalent parameter matrix can greatly simplify the relation of the effective moduli to the internal structure of the composites. For instance, a three-phase composite (fiber/interphase/matrix) can be equivalent to a two-phase composite (equivalent-fiber/matrix), if only the values of $\boldsymbol{\eta}$ are the same. Let $\mathbf{L}_{\mathrm{F}}$ denote the electroelastic moduli of the equivalent fiber in contrast to $\mathbf{L}_{\mathrm{f}}$ of the fiber in the three-phase composite, $\boldsymbol{\eta}^{(2)}$ and $\boldsymbol{\eta}^{(3)}$ denote $\boldsymbol{\eta}$ for a two-phase and a three-phase composite, respectively, and

$\boldsymbol{\eta}^{(2)}=\left(\mathbf{L}_{\mathrm{F}}+\mathbf{L}_{\mathrm{m}}\right)^{-1}\left(\mathbf{L}_{\mathrm{m}}-\mathbf{L}_{\mathrm{F}}\right)=\boldsymbol{\eta}^{(3)}$

Then $\mathbf{L}_{\mathrm{F}}$ is determined by

$\mathbf{L}_{\mathrm{F}}=\mathbf{L}_{\mathrm{m}}\left(\mathbf{I}-\boldsymbol{\eta}^{(3)}\right)\left(\mathbf{I}+\boldsymbol{\eta}^{(3)}\right)^{-1}$

It is worth noting that $\mathbf{L}_{\mathrm{F}}$ coincides with the effective moduli $\mathbf{L}_{\mathrm{e}}$ calculated by the generalized self-consistent method (Eq. (40)) for $\lambda=1$. The validity and accuracy of such an equivalent relation will be verified by high-order numerical results in Section 6.3.

\section{Numerical examples and discussions}

In the following, several typical two-phase and threephase piezoelectric composites are taken for numerical examples. The properties of the constituents are listed in Table 1.

\subsection{Convergence analysis}

First examine the convergence and accuracy of the present method. Consider two doubly periodic fiber arrays: hexagonal array and square array with $\lambda=0.6$. The variations of the effective electroelastic moduli $\left(C_{44}^{\mathrm{e}}, e_{15}^{\mathrm{e}}, \kappa_{11}^{\mathrm{e}}\right)$ with the eigenfunction expansion term number $N$ are listed in Table 2 for a two-phase composite (PZT/Epoxy) and in Table 3 for a three-phase composite (Glass/PZT/ Epoxy, $R_{0} / R=2 / 3$ ), respectively. To the best of our knowledge, neither analytical nor numerical usable results of the anti-plane electroelastic moduli of three-phase composites with doubly periodic array of coated fibers were reported. Hence only for the two-phase composite (PZT/ Epoxy), a comparison with finite element results (Berger et al., 2006; Pettermann and Suresh, 2000) is listed in Table 2 for a square fiber array and a hexagonal fiber array. From Tables 2 and 3, a good agreement and rapid convergence of the present results are observed. It is also noticed that the present first-order approximation formula $(N=1)$ possesses a good engineering accuracy and can serve as a convenient and efficient engineering closed-form formula. A further discussion refers to Fig. 3.

Table 1

Electroelastic material properties.

\begin{tabular}{llllll}
\hline & Hollow & Epoxy & Glass & BaTiO $_{3}$ & PZT \\
\hline$C_{44}(\mathrm{GPa})$ & 0 & 1.8 & 29.6 & 43.86 & 25.696 \\
$e_{15}\left(\mathrm{C} / \mathrm{m}^{2}\right)$ & 0 & 0 & 0 & 11.4 & 9.35 \\
$\kappa_{11}(\mathrm{nF} / \mathrm{m})$ & 0.00886 & 0.0372 & 0.0566 & 12.8 & 4.065 \\
\hline
\end{tabular}


Table 2

Variation of the effective electroelastic moduli $\left(C_{44}^{\mathrm{e}}, e_{15}^{\mathrm{e}}, \kappa_{11}^{\mathrm{e}}\right)$ with the term number $N$ of the eigenfunction expansion and a comparison with the finite element results (Berger et al., 2006; Pettermann and Suresh, 2000), for a two-phase composite (PZT/Epoxy) with PZT volume fraction $\lambda=0.6$.

\begin{tabular}{|c|c|c|c|c|c|c|}
\hline \multirow[t]{2}{*}{$N$} & \multicolumn{3}{|c|}{ Square array } & \multicolumn{3}{|c|}{ Hexagonal array } \\
\hline & $C_{44}^{\mathrm{e}}(\mathrm{GPa})$ & $e_{15}^{\mathrm{e}}\left(\mathrm{C} / \mathrm{m}^{2}\right)$ & $\kappa_{11}^{\mathrm{e}}(\mathrm{nF} / \mathrm{m})$ & $C_{44}^{\mathrm{e}}(\mathrm{GPa})$ & $e_{15}^{\mathrm{e}}\left(\mathrm{C} / \mathrm{m}^{2}\right)$ & $\kappa_{11}^{\mathrm{e}}(\mathrm{nF} / \mathrm{m})$ \\
\hline 1 & 6.989 & 0.05617 & 0.1657 & 6.419 & 0.04392 & 0.1493 \\
\hline 3 & 6.689 & 0.05209 & 0.1579 & 6.322 & 0.04224 & 0.1466 \\
\hline 5 & 6.672 & 0.05192 & 0.1575 & 6.324 & 0.04232 & 0.1467 \\
\hline 7 & 6.673 & 0.05195 & 0.1576 & 6.324 & 0.04232 & 0.1467 \\
\hline 9 & 6.673 & 0.05196 & 0.1576 & 6.324 & 0.04232 & 0.1467 \\
\hline FEM $^{\mathrm{a}}$ & 6.683 & 0.05204 & 0.1577 & 6.328 & 0.04244 & 0.1469 \\
\hline FEM $^{\mathrm{b}}$ & 6.643 & 0.05154 & 0.1571 & 6.298 & 0.04221 & 0.1469 \\
\hline
\end{tabular}

a Refer to Pettermann and Suresh (2000).

b Refer to Berger et al. (2006).

Table 3

Variation of the effective electroelastic moduli $\left(C_{44}^{\mathrm{e}}, e_{15}^{\mathrm{e}}, \kappa_{11}^{\mathrm{e}}\right)$ with the term number $N$ of the eigenfunction expansion, for a three-phase composite (Glass/PZT/ Epoxy) with a total volume fraction of Glass and PZT $\lambda=0.6$, and a relative radius of glass fiber $R_{0} / R=2 / 3$.

\begin{tabular}{|c|c|c|c|c|c|c|}
\hline \multirow[t]{2}{*}{$N$} & \multicolumn{3}{|c|}{ Square array } & \multicolumn{3}{|c|}{ Hexagonal array } \\
\hline & $C_{44}^{\mathrm{e}}(\mathrm{GPa})$ & $e_{15}^{\mathrm{e}}\left(\mathrm{C} / \mathrm{m}^{2}\right)$ & $\kappa_{11}^{\mathrm{e}}(\mathrm{nF} / \mathrm{m})$ & $C_{44}^{\mathrm{e}}(\mathrm{GPa})$ & $e_{15}^{\mathrm{e}}\left(\mathrm{C} / \mathrm{m}^{2}\right)$ & $\kappa_{11}^{\mathrm{e}}(\mathrm{nF} / \mathrm{m})$ \\
\hline 1 & 6.755 & 0.06127 & 0.1563 & 6.231 & 0.04855 & 0.1420 \\
\hline 3 & 6.477 & 0.05678 & 0.1495 & 6.141 & 0.04676 & 0.1397 \\
\hline 5 & 6.462 & 0.05658 & 0.1492 & 6.144 & 0.04684 & 0.1398 \\
\hline 7 & 6.462 & 0.05661 & 0.1492 & 6.144 & 0.04684 & 0.1398 \\
\hline 9 & 6.462 & 0.05662 & 0.1492 & 6.144 & 0.04684 & 0.1398 \\
\hline
\end{tabular}

\subsection{Comparison between different solutions}

To further discuss the accuracy of the first-order approximation, and the effect of the fiber distribution on the effective electroelastic moduli $\left(C_{44}^{\mathrm{e}}, e_{15}^{\mathrm{e}}, \kappa_{11}^{\mathrm{e}}\right)$, take the three-phase composite (Glass/PZT/Epoxy, $R_{0} / R=2 / 3$ ) as an example. A comparison of the present first-order approximation (EEVM, First-order) with the results of the generalized self-consistent method (GSCM) and the present high-order numerical solution (EEVM, High-order) is depicted in Fig. 3, where EEVM: eigenfunction expansionvariational method, SQU: square fiber array and HEX: hexagonal fiber array. The maximum inclusion volume fraction $\lambda$ is 0.785 for a square array, and 0.907 for a hexagonal array, while $\lambda$ can reach 1 for the GSCM because it refers to an idealized even fiber distribution. It is worth noting that in the GSCM the effective electroelastic moduli for $\lambda=1$ correspond to the moduli of the equivalent fiber of the fiber and interphase, which is mentioned in Eq. (42) and will be discussed in Subsection 6.3.

It is seen from Fig. 3 that the effective electroelastic moduli estimated by the GSCM are closer to those by present EEVM for the hexagonal array, and the present estimations for the square array are larger than both of them. For a small (dilute) volume fraction of inclusions, different solutions give almost identical results, which shows interaction between inclusions is weak. However, for a large inclusion volume fraction $\lambda$, the difference between the solutions becomes larger and larger with the increase of $\lambda$, and the difference is significant especially when $\lambda$ approaches to an extreme value. This fact reflects the interaction between inclusions becomes strong and strongly depends on fiber distribution, when fibers are close together.

Fig. 3 also shows that the first-order approximation formula possesses a good accuracy when $\lambda<0.7$ for a square array and $\lambda<0.8$ for a hexagonal array. For $\lambda$ out of this range, the high-order numerical solutions are needed to obtain accurate results.

\subsection{The equivalency between three-phase and two-phase composites by $\boldsymbol{\eta}$}

In the first-order approximation formula (37) and the generalized self-consistent formula (40), the equivalent parameter matrix $\boldsymbol{\eta}$ is extracted. This matrix can reflect the overall influence of the electroelastic properties of the fiber and interphase on the effective moduli, and by which a three-phase composite can be equivalent to a two-phase composite. For high-order numerical results, does such an equivalent relation still exist? To answer this question, take two kinds of three-phase piezoelectric composites for examples: Glass/PZT/Epoxy with a square fiber array and Hollow/PZT/BaTiO 3 with a hexagonal fiber array. The moduli of the equivalent fiber in the corresponding equivalent two-phase composite are calculated by Eq. (42) for different interphase thickness. The high-order numerical results of the effective electroelastic moduli of the three-phase and corresponding equivalent two-phase composites versus the relative interphase thickness for different volume fraction $\lambda$ are depicted in Fig. 4 for the Glass/ $\mathrm{PZT} /$ Epoxy and in Fig. 5 for the Hollow/PZT/BaTiO respectively. 


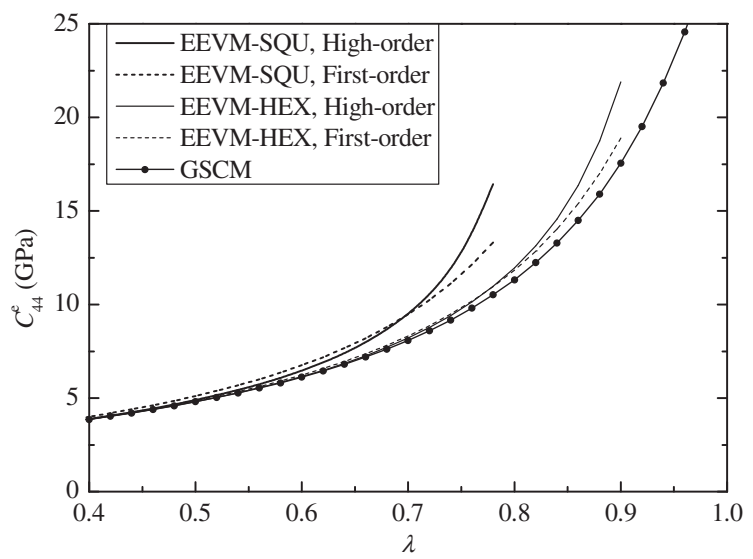

(a)

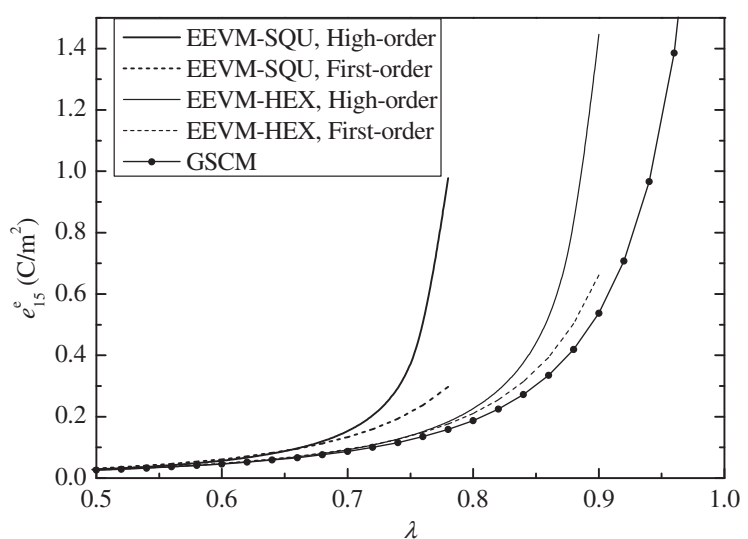

(b)

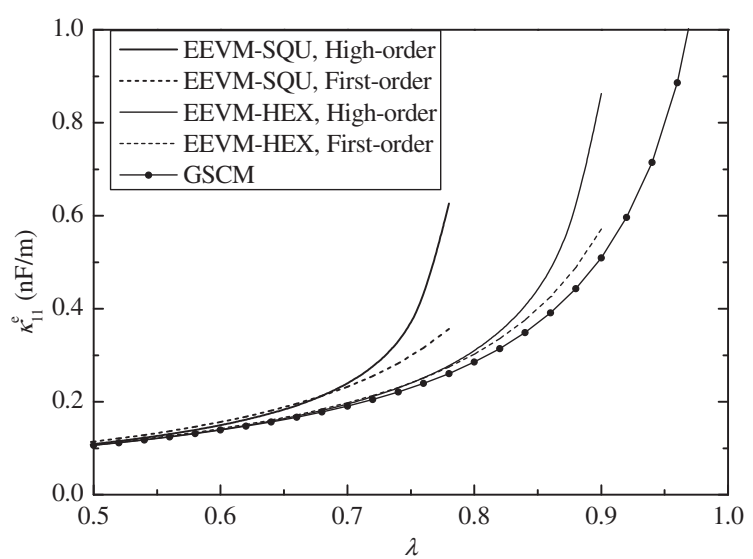

(c)

Fig. 3. A comparison of the present first-order approximation and highorder numerical solution for a square fiber array and a hexagonal fiber array, with the generalized self-consistent estimation, for a three-phase composite (Glass/PZT/Epoxy, $R_{0} / R=2 / 3$ ): (a) effective shear modulus $C_{44}^{\mathrm{e}}$; (b) effective piezoelectric modulus $e_{15}^{\mathrm{e}}$; (c) effective dielectric modulus $\kappa_{11}^{\mathrm{e}}$.

From Figs. 4 and 5, it is seen that the effective moduli of the three-phase composites and the corresponding equivalent two-phase composites agree well even when volume

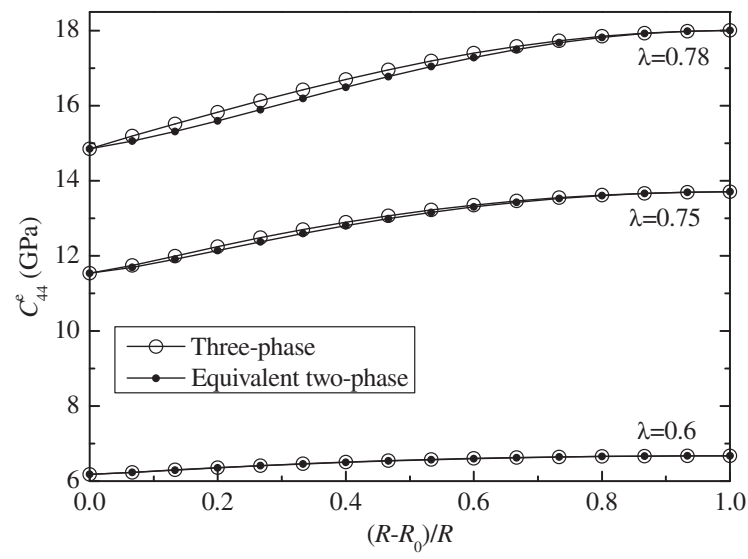

(a)

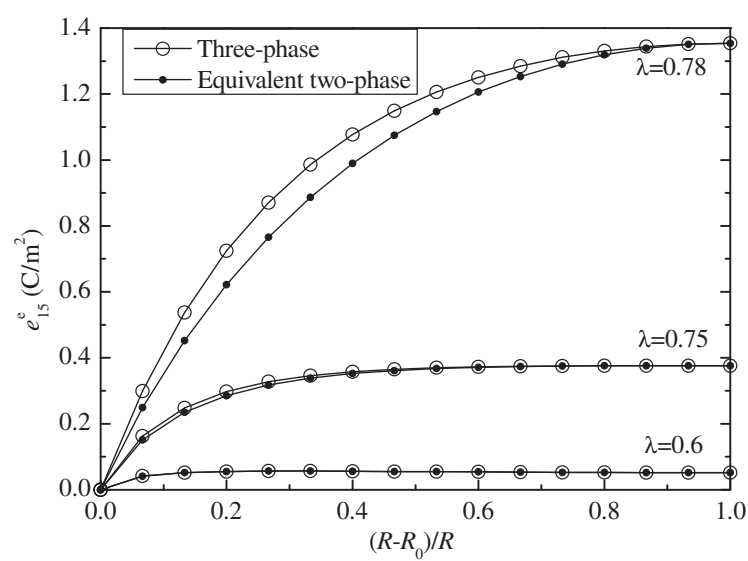

(b)

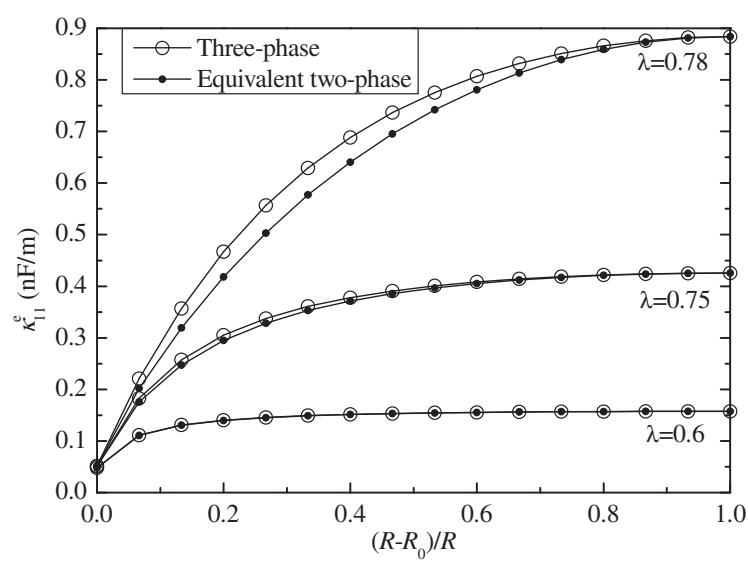

(c)

Fig. 4. For a three-phase composite (Glass/PZT/Epoxy) with a square fiber array, the high-order numerical results of the effective electroelastic moduli of the three-phase and corresponding equivalent two-phase composites versus the relative interphase thickness $\left(R-R_{0}\right) / R$ for different volume fraction $\lambda$ : (a) effective shear modulus $C_{44}^{\mathrm{e}}$; (b) effective piezoelectric modulus $e_{15}^{\mathrm{e}}$; (c) effective dielectric modulus $\kappa_{11}^{\mathrm{e}}$.

fraction $\lambda$ approaches 0.75 for the square array and 0.90 for hexagonal array. This fact indicates that the equivalence between a three-phase composite and the corresponding equivalent two-phase composite by $\boldsymbol{\eta}$ is 


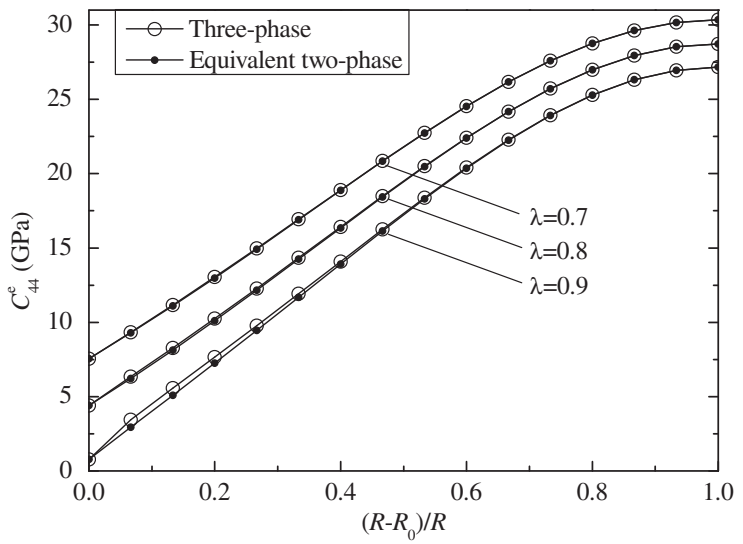

(a)

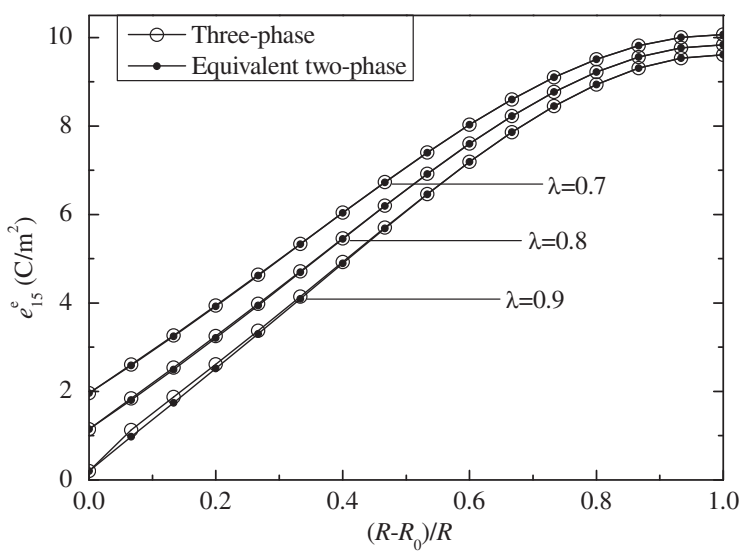

(b)

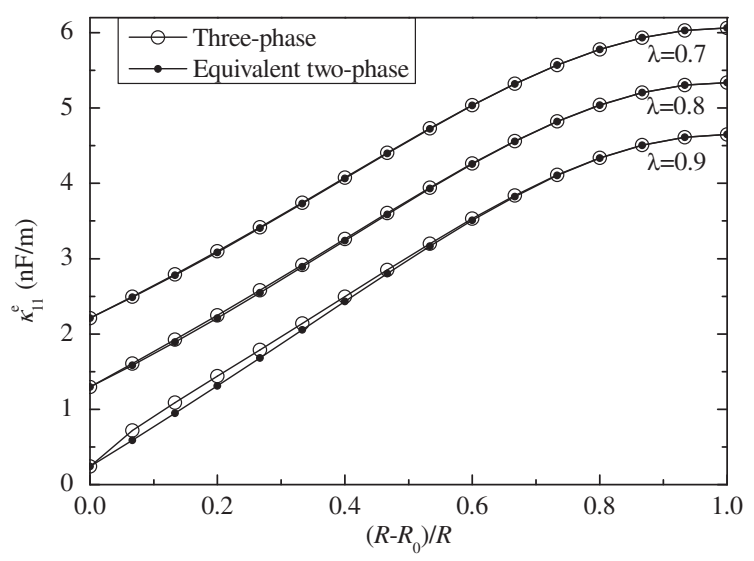

(c)

Fig. 5. For a three-phase composite (Hollow/PZT/BaTiO $)$ with a hexagonal fiber array, the high-order numerical results of the effective electroelastic moduli of the three-phase and corresponding equivalent two-phase composites versus the relative interphase thickness $\left(R-R_{0}\right) / R$ for different volume fraction $\lambda$ : (a) effective shear modulus $C_{44}^{\mathrm{e}}$; (b) effective piezoelectric modulus $e_{15}^{\mathrm{e}}$; (c) effective dielectric modulus $\kappa_{11}^{\mathrm{e}}$.

reasonable, though it is approximate nevertheless. Therefore, $\boldsymbol{\eta}$ can indeed reflect a combined effect of the fiber and interphase properties on the effective moduli with a good accuracy and can greatly simplify the relation of effective moduli to the internal structure of a composite.

It is also seen from Figs. 4(b) and 5(b) that, with the increase in the relative thickness $\left(R-R_{0}\right) / R$ of the piezoelectric interphase, the effective piezoelectric modulus $e_{15}^{\mathrm{e}}$ increases dramatically, then levels out and finally becomes nearly saturated. This fact is useful for design optimization of the three-phase piezoelectric composites (Beckert et al., 2001; Marcheselli and Venkatesh, 2008): if only the relative radius $R_{0} / R$ of the glass fiber is less than 0.4 , the glass fiber in the Glass/PZT/Epoxy provides the mechanical support meanwhile the effective piezoelectric modulus is without great decrease; similarly, if only $R_{0} /$ $R<0.2$, the hollow in the Hollow/PZT/BaTiO 3 reduces the density meanwhile the effective piezoelectric modulus is without great decrease.

\section{Conclusions}

A new variational functional for a unit cell is constructed to deal with the anti-plane electroelastic behavior of three-phase piezoelectric composites with doubly periodic microstructures. Then, by combining with the eigenfunction expansions of the complex potentials, an eigenfunction expansion-variational method based on a unit cell is developed. The numerical results of the effective electroelastic moduli show a rapid convergence of the present method. By introducing a simple scalar parameter $\alpha$, a unified first-order approximation formula is also provided, which can cover square and hexagonal fiber distributions, and can also cover the generalized self-consistent estimation. Compared with the present high-order numerical results, the first-order approximation possesses a good accuracy when $\lambda<0.7$ for a square array and $\lambda<0.8$ for a hexagonal array, thus can serve as a convenient and efficient engineering closed-form formula.

An equivalent parameter matrix $\boldsymbol{\eta}$ is extracted in the unified first-order approximation formula. The matrix $\boldsymbol{\eta}$ can reflect the overall influence of the fiber and interphase properties on the effective electroelastic moduli of the three-phase composites, and can greatly simplify the complicated relation of the effective moduli to the internal structure of a composite. And the validity and accuracy of the matrix $\boldsymbol{\eta}$ are verified by the high-order numerical results.

The numerical examples reveal good designability of the three-phase composites as multifunctional composites. For a Glass/PZT/Epoxy composite, if only the relative radius $R_{0} / R$ of the glass fiber is less than 0.4 , the glass fiber provides the mechanical support meanwhile the effective piezoelectric modulus is without great decrease; similarly, for a Hollow $/ \mathrm{PZT} / \mathrm{BaTiO}_{3}$ composite, if only $R_{0} / R<0.2$, the hollow reduces the density without greatly decreasing the effective piezoelectric modulus.

\section{Acknowledgements}

The work is supported by the National Natural Science Foundation of China under Grant NNSFC 10972020, and 
the Fundamental Research Funds for the Central Universities.

Appendix A. Derivations of Eqs. (22)-(25)

The Substitution of Eqs. (20a)-(20c) into Eqs. (19a) and (19c) yields the expansions of the generalized displacement $\mathbf{w}$ and generalized resultant force $\mathbf{T}$ in the fiber, coating/interphase and matrix regions, respectively,

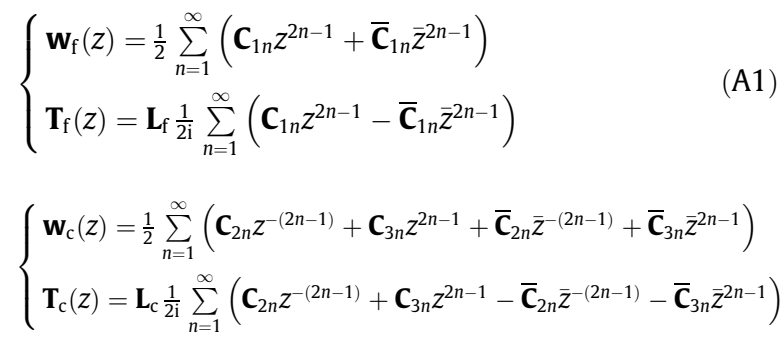

$$
\left\{\begin{array}{l}
\mathbf{w}_{\mathrm{m}}(z)=\frac{1}{2} \sum_{n=1}^{\infty}\left(\mathbf{C}_{4 n} z^{-(2 n-1)}+\mathbf{C}_{5 n} z^{2 n-1}+\overline{\mathbf{C}}_{4 n} \bar{z}^{-(2 n-1)}+\overline{\mathbf{C}}_{5 n} \bar{z}^{2 n-1}\right) \\
\mathbf{T}_{\mathrm{m}}(z)=\mathbf{L}_{\mathrm{m}} \frac{1}{2 \mathrm{i}} \sum_{n=1}^{\infty}\left(\mathbf{C}_{4 n} z^{-(2 n-1)}+\mathbf{C}_{5 n} z^{2 n-1}-\overline{\mathbf{C}}_{4 n} \bar{z}^{-(2 n-1)}-\overline{\mathbf{C}}_{5 n} \bar{z}^{2 n-1}\right)
\end{array}\right.
$$

At the fiber-coating interface, $z \bar{z}=R_{0}^{2}$, substituting Eqs. (A1) and (A2) into interface condition (21a) and replacing $\bar{z}$ with $R_{0}^{2} z^{-1}$, one obtains

$$
\begin{aligned}
& \left\{\begin{array}{l}
\mathbf{C}_{1 n}=\mathbf{C}_{3 n}+\overline{\mathbf{C}}_{2 n} R_{0}^{-2(2 n-1)} \\
\mathbf{L}_{\mathrm{f}} \mathbf{C}_{1 n}=\mathbf{L}_{\mathrm{c}}\left(\mathbf{C}_{3 n}-\overline{\mathbf{C}}_{2 n} R_{0}^{-2(2 n-1)}\right)
\end{array}\right. \\
& \left\{\begin{array}{l}
\mathbf{C}_{3 n}+\overline{\mathbf{C}}_{2 n} R^{-2(2 n-1)}=\mathbf{C}_{5 n}+\overline{\mathbf{C}}_{4 n} R^{-2(2 n-1)} \\
\mathbf{L}_{\mathrm{c}}\left(\mathbf{C}_{3 n}-\overline{\mathbf{C}}_{2 n} R^{-2(2 n-1)}\right)=\mathbf{L}_{\mathrm{m}}\left(\mathbf{C}_{5 n}-\overline{\mathbf{C}}_{4 n} R^{-2(2 n-1)}\right)
\end{array}\right.
\end{aligned}
$$

Solving Eq. (A6), one obtains the relation between $\mathbf{C}_{2 n}$ and $\mathbf{C}_{3 n}$,

$\overline{\mathbf{C}}_{2 n}=\boldsymbol{\eta}_{\mathrm{fc}} R_{0}^{2(2 n-1)} \mathbf{C}_{3 n}$

where $\boldsymbol{\eta}_{\mathrm{fc}}=\left(\mathbf{L}_{\mathrm{f}}+\mathbf{L}_{\mathrm{c}}\right)^{-1}\left(\mathbf{L}_{\mathrm{c}}-\mathbf{L}_{\mathrm{f}}\right)$. Substituting Eq. (A8) into (A7), and then solving (A7), one obtains the relation between $\mathbf{C}_{4 n}$ and $\mathbf{C}_{5 n}$, that is, Eqs. (22)-(25).

\section{Appendix B. Derivations of Eqs. (37)-(40)}

When only one term of the eigenfunction expansion is taken, $N=1$, form Eqs. (36) and (31b) a first-order approximation formula is obtained as:

$$
\mathbf{L}_{\mathrm{e}}=\mathbf{L}_{22}^{\mathrm{e}}=\frac{1}{V}\left(\sum_{s} \mathbf{T}_{(1)}^{s+} p_{2}^{s}\right)\left(\mathbf{A}_{11}^{-1}\right)\left(\sum_{s} \mathbf{T}_{(1)}^{s+} p_{2}^{s}\right)
$$

where

$$
\begin{aligned}
\mathbf{A}_{11}= & \sum_{S} \int_{\partial V_{s}^{+}}\left(\mathbf{t}_{(1)}^{s+}\right)^{\mathrm{T}}\left(\mathbf{w}_{(1)}^{S+}-\mathbf{w}_{(1)}^{S-}\right) d S \\
& -\sum_{S} \int_{\partial V_{s}^{+}}\left(\mathbf{w}_{(1)}^{S-}\right)^{\mathrm{T}}\left(\mathbf{t}_{(1)}^{S+}+\mathbf{t}_{(1)}^{S-}\right) d S
\end{aligned}
$$

$$
\left\{\begin{array}{l}
\sum_{n=1}^{\infty}\left(\mathbf{C}_{1 n} z^{2 n-1}+\overline{\mathbf{C}}_{1 n} R_{0}^{2(2 n-1)} z^{-(2 n-1)}\right)=\sum_{n=1}^{\infty}\left[\left(\mathbf{C}_{3 n}+\overline{\mathbf{C}}_{2 n} R_{0}^{-2(2 n-1)}\right) z^{2 n-1}+\left(\mathbf{C}_{2 n}+\overline{\mathbf{C}}_{3 n} R_{0}^{2(2 n-1)}\right) z^{-(2 n-1)}\right] \\
\mathbf{L}_{\mathrm{f}} \sum_{n=1}^{\infty}\left(\mathbf{C}_{1 n} z^{2 n-1}-\overline{\mathbf{C}}_{1 n} R_{0}^{2(2 n-1)} z^{-(2 n-1)}\right)=\mathbf{L}_{\mathrm{c}} \sum_{n=1}^{\infty}\left[\left(\mathbf{C}_{3 n}-\overline{\mathbf{C}}_{2 n} R_{0}^{-2(2 n-1)}\right) z^{2 n-1}+\left(\mathbf{C}_{2 n}-\overline{\mathbf{C}}_{3 n} R_{0}^{2(2 n-1)}\right) z^{-(2 n-1)}\right]
\end{array}\right.
$$

Similarly, at the coating-matrix interface, $z \bar{z}=R^{2}$, substituting Eqs. (A2) and (A3) into interface condition (21b) and replacing $\bar{z}$ with $R^{2} z^{-1}$, one obtains
Noting Eq. (23), let

$$
\begin{aligned}
\boldsymbol{\eta}= & \boldsymbol{\eta}_{1}=\left[\mathbf{I}+\left(\mathbf{I}+\mathbf{L}_{\mathrm{c}}^{-1} \mathbf{L}_{\mathrm{m}}\right)^{-1} \boldsymbol{\eta}_{\mathrm{fc}}\left(\mathbf{L}_{\mathrm{c}}^{-1} \mathbf{L}_{\mathrm{m}}-\mathbf{I}\right)\left(R_{0} / R\right)^{2}\right]^{-1} \\
& \cdot\left[\left(\mathbf{I}+\mathbf{L}_{\mathrm{c}}^{-1} \mathbf{L}_{\mathrm{m}}\right)^{-1} \boldsymbol{\eta}_{\mathrm{fc}}\left(\mathbf{I}+\mathbf{L}_{\mathrm{c}}^{-1} \mathbf{L}_{\mathrm{m}}\right)\left(R_{0} / R\right)^{2}+\boldsymbol{\eta}_{\mathrm{cm}}\right]
\end{aligned}
$$

$$
\left\{\begin{array}{l}
\sum_{n=1}^{\infty}\left[\left(\mathbf{C}_{3 n}+\overline{\mathbf{C}}_{2 n} R^{-2(2 n-1)}\right) z^{2 n-1}+\left(\mathbf{C}_{2 n}+\overline{\mathbf{C}}_{3 n} R^{2(2 n-1)}\right) z^{-(2 n-1)}\right]=\sum_{n=1}^{\infty}\left[\left(\mathbf{C}_{5 n}+\overline{\mathbf{C}}_{4 n} R^{-2(2 n-1)}\right) z^{2 n-1}+\left(\mathbf{C}_{4 n}+\overline{\mathbf{C}}_{5 n} R^{2(2 n-1)}\right) z^{-(2 n-1)}\right] \\
\mathbf{L}_{\mathrm{c}} \sum_{n=1}^{\infty}\left[\left(\mathbf{C}_{3 n}-\overline{\mathbf{C}}_{2 n} R^{-2(2 n-1)}\right) z^{2 n-1}+\left(\mathbf{C}_{2 n}-\overline{\mathbf{C}}_{3 n} R^{2(2 n-1)}\right) z^{-(2 n-1)}\right]=\mathbf{L}_{\mathrm{m}} \sum_{n=1}^{\infty}\left[\left(\mathbf{C}_{5 n}-\overline{\mathbf{C}}_{4 n} R^{-2(2 n-1)}\right) z^{2 n-1}+\left(\mathbf{C}_{4 n}-\overline{\mathbf{C}}_{5 n} R^{2(2 n-1)}\right) z^{-(2 n-1)}\right]
\end{array}\right.
$$

Comparing the coefficients of the same order of $z$ in Eqs. (A4) and (A5), four sets of equations with respect to the five sets of unknown complex coefficients are obtained as: and then the eigenfunction expansions of the generalized stresses, displacement, boundary stress and resultant force (Eq. (30)) can be rewritten as follows: 


$$
\left\{\begin{array}{l}
\tau_{1}^{(1)}=\frac{-1}{2 \mathrm{i}} \mathbf{L}_{\mathrm{m}}\left[\boldsymbol{\eta} R^{2}\left(\bar{z}^{-2}-z^{-2}\right)\right] \\
\tau_{2}^{(1)}=\frac{1}{2} \mathbf{L}_{\mathrm{m}}\left[\boldsymbol{\eta} R^{2}\left(\bar{z}^{-2}+z^{-2}\right)\right] \\
\left.\mathbf{w}^{(1)}=\frac{1}{2 \mathrm{i}} \mathbf{I}(z-\bar{z})+\boldsymbol{\eta} R^{2}\left(\bar{z}^{-1}-z^{-1}\right)\right] \\
\mathbf{t}^{(1)}=\boldsymbol{\tau}^{(1)} \mathbf{n} \\
\mathbf{T}^{(1)}=-\frac{1}{2} \mathbf{L}_{\mathrm{m}}\left[\mathbf{I}(z+\bar{z})-\boldsymbol{\eta} R^{2}\left(\bar{z}^{-1}+z^{-1}\right)\right]_{\mathrm{A}}^{\mathrm{B}}
\end{array}\right.
$$

For a square array shown in Fig. 1(c),

$\mathbf{p}^{1}=R \sqrt{\frac{\pi}{\lambda}}\{1,0\}, \quad \mathbf{p}^{2}=R \sqrt{\frac{\pi}{\lambda}}\{0,1\}$

and the summations and integrations in Eqs. (B1) and (B2) can be simplified as:

$$
\begin{aligned}
& \mathbf{A}_{11}=-\frac{\pi}{\lambda} \mathbf{L}_{\mathrm{m}}+(2-2 \pi) \mathbf{L}_{\mathrm{m}} \boldsymbol{\eta}+2 \boldsymbol{\eta}^{\mathrm{T}} \mathbf{L}_{\mathrm{m}}+\frac{4 \lambda}{\pi} \boldsymbol{\eta}^{\mathrm{T}} \mathbf{L}_{\mathrm{m}} \boldsymbol{\eta} \\
& \sum_{s} \mathbf{T}_{(1)}^{s+} p_{2}^{s}=\mathrm{i}\left(\frac{\pi}{\lambda} \mathbf{L}_{\mathrm{m}}-2 \mathbf{L}_{\mathrm{m}} \boldsymbol{\eta}\right)
\end{aligned}
$$

For a hexagonal array shown in Fig. 1(b),

$$
\begin{aligned}
& \mathbf{p}^{1}=R \sqrt{\frac{2 \pi}{\sqrt{3} \lambda}}\{1,0\}, \quad \mathbf{p}^{2}=R \sqrt{\frac{2 \pi}{\sqrt{3} \lambda}}\left\{\frac{1}{2}, \frac{\sqrt{3}}{2}\right\}, \\
& \mathbf{p}^{3}=R \sqrt{\frac{2 \pi}{\sqrt{3} \lambda}}\left\{-\frac{1}{2}, \frac{\sqrt{3}}{2}\right\}
\end{aligned}
$$

and the summations and integrations in Eqs. (B1) and (B2) can be simplified as:

$$
\mathbf{A}_{11}=-\frac{\pi}{\lambda} \mathbf{L}_{\mathrm{m}}+\left(\frac{3 \sqrt{3}}{2}-2 \pi\right) \mathbf{L}_{\mathrm{m}} \boldsymbol{\eta}+\frac{3 \sqrt{3}}{2} \boldsymbol{\eta}^{\mathrm{T}} \mathbf{L}_{\mathrm{m}}+\frac{27 \lambda}{4 \pi} \boldsymbol{\eta}^{\mathrm{T}} \mathbf{L}_{\mathrm{m}} \boldsymbol{\eta}
$$

$\sum_{s} \mathbf{T}_{(1)}^{s+} p_{2}^{s}=\mathrm{i}\left(\frac{\pi}{\lambda} \mathbf{L}_{\mathrm{m}}-\frac{3 \sqrt{3}}{2} \mathbf{L}_{\mathrm{m}} \boldsymbol{\eta}\right)$

Combining with Eqs. (B6), (B7) and (B9), (B10), and noting $\boldsymbol{\eta}^{\mathrm{T}} \mathbf{L}_{\mathrm{m}}=\mathbf{L}_{\mathrm{m}} \boldsymbol{\eta}$, the first-order approximation formula (B1) can be written as a unified expression, that is, Eq. (37).

When $\alpha=\pi$, Eq. (37) can be reduced as:

$$
\begin{aligned}
\mathbf{L}_{\mathrm{e}} & =\mathbf{L}_{\mathrm{m}}(\pi \mathbf{I}-\pi \lambda \boldsymbol{\eta})^{2}\left(\pi^{2} \mathbf{I}-\pi^{2} \lambda^{2} \boldsymbol{\eta}^{2}\right)^{-1} \\
& =\mathbf{L}_{\mathrm{m}}(\mathbf{I}-\lambda \boldsymbol{\eta})^{2}(\mathbf{I}-\lambda \boldsymbol{\eta})^{-1}(\mathbf{I}+\lambda \boldsymbol{\eta})^{-1} \\
& =\mathbf{L}_{\mathrm{m}}(\mathbf{I}-\lambda \boldsymbol{\eta})(\mathbf{I}+\lambda \boldsymbol{\eta})^{-1}
\end{aligned}
$$

which coincides with the result of the generalized self-consistent method (Jiang and Cheung, 2001; Jiang et al., 2001). In this case, the fiber distribution can be regarded as an idealized even distribution, whose configuration is depicted in Hu and Weng (2000), where the shapes of the double-cell and inclusion are both circular. Thus, $\alpha$ is a parameter reflecting the distribution of fibers, and Eq. (39) can be rewritten as:

$$
\alpha= \begin{cases}2 & \text { for square array } \\
3 \sqrt{3} / 2 & \text { for hexagonal array } \\
\pi & \text { for idealized even distribution }\end{cases}
$$

\section{References}

Beckert, W., Kreher, W., Braue, W., Ante, M., 2001. Effective properties of composites utilising fibres with a piezoelectric coating. J. Eur. Ceram. Soc. 21, 1455-1458.

Berger, H., Kari, S., Gabbert, U., Rodriguez-Ramos, R., Bravo-Castillero, J., Guinovart-Diaz, R., Sabina, F.J., Maugin, G.A., 2006. Unit cell models of piezoelectric fiber composites for numerical and analytical calculation of effective properties. Smart Mater. Struct. 15, 451-458.

Bravo-Castillero, J., Guinovart-Díaz, R., Sabina, F.J., Rodríguez-Ramos, R., 2001. Closed-form expressions for the effective coefficients of a fiberreinforced composite with transversely isotropic constituents. II: Piezoelectric and square symmetry. Mech. Mater. 33, 237-248.

Chan, H.L.W., Unsworth, J., 1989. Simple model for piezoelectric ceramic/ polymer 1-3 composites used in ultrasonic transducer applications. IEEE Trans. Ultrason. Ferroelec. Freq. Contr. 36, 434-441.

Dinzart, F., Sabar, H., 2009. Electroelastic behavior of piezoelectric composites with coated reinforcements: Micromechanical approach and applications. Int. J. Solids Struct. 46, 3556-3564.

Guinovart-Díaz, R., Rodríguez-Ramos, R., Bravo-Castillero, J., Sabina, F.J., Camacho-Montes, H., 2008. Electro-mechanical moduli of threephase fiber composites. Mater. Lett. 62, 2385-2387.

Hashemi, R., Weng, G.J., Kargarnovin, M.H., Shodja, H.M., 2010. Piezoelectric composites with periodic multi-coated inhomogeneities. Int. J. Solids Struct. 47, 2893-2904.

Hu, G.K., Weng, G.J., 2000. The connections between the double-inclusion model and the Ponte Castaneda-Willis, Mori-Tanaka, and KusterToksoz models. Mech. Mater. 32, 495-503.

Jiang, C.P., Cheung, Y.K., 2001. An exact solution for the three-phase piezoelectric cylinder model under antiplane shear and its applications to piezoelectric composites. Int. J. Solids Struct. 38, 4777-4796.

Jiang, C.P., Tong, Z.H., Cheung, Y.K., 2001. A generalized self-consistent method for piezoelectric fiber reinforced composites under antiplane shear. Mech. Mater. 33, 295-308.

Koutsawa, Y., Biscani, F., Belouettar, S., Nasser, H., Carrera, E., 2010. Multicoating inhomogeneities approach for the effective thermo-electroelastic properties of piezoelectric composite materials. Compos. Struct. 92, 964-972.

Lin, Y., Sodano, H.A., 2008. Concept and model of a piezoelectric structural fiber for multifunctional composites. Compos. Sci. Technol. 68, 19111918.

Lin, Y., Sodano, H.A., 2010. A double inclusion model for multiphase piezoelectric composites. Smart Mater. Struct. 19, 035003.

Marcheselli, C., Venkatesh, T.A., 2008. Electromechanical response of 1-3 piezoelectric composites with hollow fibers. Appl. Phys. Lett. 93, 022903.

Nemat-Nasser, S., Hori, M., 1999. Micromechanics: Overall Properties of Heterogeneous Materials. Elsevier, Netherlands.

Pettermann, H.E., Suresh, S., 2000. A comprehensive unit cell model: A study of coupled effects in piezoelectric 1-3 composites. Int. J. Solids Struct. 37, 5447-5464.

Rodríguez-Ramos, R., Yan, P., López-Realpozo, J.C., Guinovart-Díaz, R., Bravo-Castillero, J., Sabina, F.J., Jiang, C.P., 2010. Two analytical models for the study of periodic fibrous elastic composite with different unit cells. Compos. Struct. 93, 709-714.

Shen, M.H., Chen, F.M., Chen, S.N., Hung, S.Y., 2009. Piezoelectric study for a coated hole of quasi-polygonal shape in an infinite plate. Int. J. Eng. Sci. 47, 475-486.

Shen, M.H., Chen, S.N., Chen, F.M., 2005. Piezoelectric study on confocally multicoated elliptical inclusion. Int. J. Eng. Sci. 43, 12991312.

Smith, W.A., Shaulov, A., Auld, B.A., 1985. Tailoring the properties of composite piezoelectric materials for medical ultrasonic transducers. IEEE Ultrason. Sympos. 2, 642-647.

Sudak, L.J., 2003. Effect of an interphase layer on the electroelastic stresses within a three-phase elliptic inclusion. Int. J. Eng. Sci. 41, 1019-1039.

Sun, W., Lin, F., Hu, X., 2001. Computer-aided design and modeling of composite unit cells. Compos. Sci. Technol. 61, 289-299.

Tiersten, H.F., 1969. Linear Piezoelectric Plate Vibrations. Plenum Press, New York.

Tong, Z.H., Jiang, C.P., Lo, S.H., Cheung, Y.K., 2006. A closed form solution to the antiplane problem of doubly periodic cracks of unequal size in piezoelectric materials. Mech. Mater. 38, 269-286. 
Xia, Z., Zhang, Y., Ellyin, F., 2003. A unified periodical boundary conditions for representative volume elements of composites and applications. Int. J. Solids Struct. 40, 1907-1921.

Xu, Y.L., Lo, S.H., Jiang, C.P., Cheung, Y.K., 2007. Electroelastic behavior of doubly periodic piezoelectric fiber composites under antiplane shear. Int. J. Solids Struct. 44, 976-995.
Yang, B.-H., Gao, C.-F., 2009. Anti-plane electro-elastic fields in an infinite matrix with $\mathrm{N}$ coated-piezoelectric inclusions. Compos. Sci. Technol. 69, 2668-2674.

Yu, N., Somphone, T., 2009. The inclusion and inhomogeneity problems of electrostrictive materials with periodic microstructure. Mech. Mater. 41, 975-981. 